\title{
Bose-Einstein Condensation Studied by the Real-Time Monte Carlo Simulation in the Frame of Java Applet
}

\author{
Monika Gall, Ryszard Kutner, Andrzej Majerowski, and Dariusz Żebrowski \\ Institute of Experimental Physics, Department of Physics, Warsaw University, \\ Smyczkowa 5/7, Pl-02678 Warsaw, Poland
}

\begin{abstract}
A Monte Carlo algorithm was constructed combined with a Java applet for the simulation of statistical physics quantities characterizing noninteracting bosons within micro- and macroscales. By this approach the Bose-Einstein condensate was considered within a threedimensional isotropic harmonic oscillator in real-time. The algorithm can be used to study both the static properties of ideal bosons within other trapping potentials and the relaxation of the system to the condensate. The algorithm can be extended to cover also collisions between bosons. Conluding, our approach can be used for studying and visualizing both educational and professional problems regarding quantum statistical physics of bosonic systems.
\end{abstract}

\section{Introduction}

In educational and professional computational physics lattice gas models have attracted much attention since they can be successfully used in Monte Carlo simulations [1], particularly in real-time computer experiments. The principal goal of this paper is to show the main possibilities of our software to study, within micro- and macroscales, the noninteracting bosons. We show that it can mimic the quantum statistical physics features of particles by using standard MC simulations and a lattice gas model provided with a peculiar requirement (cf. Sec2). We trace the consequences of this mimic, mainly by simulation of the Bose-Einstein condensate (BEC), which can be treated as a macroscopic system. Since below the transition temperature the ground level is occupied by a marcoscopic amount of bosons, the situation can be considered within the macroscale in distinction from the case where the temperature exceeds the transition temperature. Here, a microscopic amount of bosons occupy each level which defines the case that should be considered within the microscale.

By macroscopic occupancy of the ground energy level we understand that the ratio $\left\langle N_{0}\right\rangle / N$ is larger than zero even at the limit where the total amount of bosons $N$ in the system (which possesses infinitely many energy levels) increases to infinity; of course, the normalization $\sum_{h=0}^{\infty}\left\langle N_{h}\right\rangle / N=1$ is always obeyed, where $\left\langle N_{h}\right\rangle, h=0,1,2, \ldots$, is the average number of bosons occupying the energy level $h$. We can say that the occupancy of any energy level is microscopic 
if $\lim _{N \rightarrow \infty}\left\langle N_{h}\right\rangle / N=0$. Of course, in our numerical experiments performed within finite systems we can observe only the finger print of macroscopic and microscopic energy level occupancies.

We hope that our MC algorithm offers a complementary possibility for studying statistical and thermal physics of Bose-Einstein condensate (i.e. its static and dynamic properties) both on educational and professional levels. Since 1995, when this exotic state of matter was experimentally observed in dilute atomic gases for the first time [23], this state is the subject of intense analytical, numerical, experimental and educational studies [4]. The present work is a direct continuation of our previous one [5] extended by including the interactive Java applet, which is a modern, platform-independent programming technology useful both for educational and professional purposes (our applet is working under the Java 2 version 1.4.1.1 or higher).

\section{Model and Simulation Procedure}

We define the model together with the algorithm for Monte Carlo simulation. The algorithm allows us to calculate relevant quantities within different statistical ensembles 6 6]. This algorithm makes possible to study both static and dynamic statistical physics quantities such as ground- and excited state occupancies of energy levels by bosons and the corresponding fluctuations, internal energy of the system, its entropy and specific heat. Thus, it provides an approach to consider thermal and statistical physics of quantum (and classical) gases in equilibrium and non-equilibrium states both on educational and professional levels. It should be noted that none of the known numerical methods has so wide possibilities. For example, by using partition functions one can calculate only equilibrium quantities 68); an alternative MC simulation of BEC for ideal bosonic gas but enclosed in a rigid box was performed in [9].

In the case of a classic lattice gas identical (point) particles are considered to be distinguishable and any number of particles in the same site can be understood as a single-particle quantum-mechanical state. However, the classical particles require in some sense an even more rafined treatment than the quantum ones since they are genuinely indistinguishable when occupying the same single-particle quantum-mechanical state but otherwise they are entirely distinguishable and can then be treated as entirely independent particles. Such particles obey the Boltzmann statistics which is quantum-mechanically incorrect but can serve, e.g., as a reference high-temperature case.

The basic feature of our lattice gas model consists in that it mimics quantum indistinguishability, where the net result of a simple interchange of two identical particles is that no new state of the whole lattice gas is obtained. In distinction from the classical lattice gas, it does not matter which particle is in a given single-particle state, but only how many particles are in this state. The simplest example considered below should make this general idea clearer and make possible the construction of an algorithm. 


\subsection{Strategy of the Algorithm}

Let us consider, for example, a lattice gas consisting of only two identical particles and call them 1 and 2, which is an auxiliary numeration which helps us to describe the idea of our algorithm, (we also consider in higher dimensions an equivalent algorithm where no auxiliary numbering of particles is necessary). We denote two lattice sites, i.e. different single-particle states as $h=0$ and $h=1$. According to quantum indistinguishability, the configuration where particle 1 is at state $h=0$ and particle 2 is at state $h=1$ is no longer counted as distinct from the configuration where these two particles are interchanged. Thus, we see the basic step of our algorithm since we can decide that particle number 1 never has a higher energy than particle number 2 (the opposite situation is disregarded). In other words, the sequence of particles is dynamically preserved during the whole simulation which defines the conservation principle which is a generalization of the Pauli exclusion principle valid for fermions or fermionic lattice gas. As it is seen, the space of states for this system is shrunk and the correlation between particles exist when the total number of particles in the system is fixed (e.g., for canonical or microcanonical ensembles). The above introduced conservation principle has already been used by us to study one-dimensional bosonic lattice gas (BLG) [10].

\subsection{Local Dynamics}

We confine our attention to spinless particles; for simplicity we assume that there is no mutual interaction between particles apart from the above introduced conservation principle which, however, can introduce correlations between them.

The initial particle configuration was randomly selected but other initial configurations are also accepted since the final equilibrium result does not depend on the choice of the initial condition.

We deal with a three-dimensional isotropic harmonic oscillator. Then each (energy) level, $h(=0,1,2, \ldots)$, of the energetic ladder is degenerated and consists of $g(h)=\frac{1}{2} h(h+3)+1$ single-particle states (sites); the energy level spacing (which is fixed) is denoted as $\Delta \varepsilon$. Every state is defined here by the state-vector $\underline{h}=\left(h_{1}, h_{2}, h_{3}\right)$, where $h_{1}, h_{2}, h_{3} \geq 0$, while $h=h_{1}+h_{2}+h_{3}$.

Note that two states can be connected here by a direct jump of a boson only if the chosen components of the initial and final states differ by \pm 1 . This configuration of states together with the above rule for jumps determine, in fact, the bosonic lattice gas defined by the three-dimensional lattice consisting of sitevectors having non-negative coordinates. As, for simplicity, particle jumps are assumed to occur only between the nearest-neighbor sites, there are no direct jumps between states belonging to the same energy level (since they would only slow down the relaxation).

From the strategy developed in Sec2.1 it follows that one and only one particle from all $N_{\underline{h}}$ ones currently occupying the given single-particle state $\underline{h}$ performs an upward jump and it is unimportant which one, since the particles are indistinguishable in the quantum-mechanical sense. We have a similar situation 
for any excited state when a downward jump is performed. The above defines the strategy of an alternative algorithm, particularly effective when energy levels are degenerated (as is the case of the three-dimensional isotropic harmonic oscillator). Namely, instead of using the above defined numbering of particles it is sufficient to choose at random a particle from an occupied (earlier chosen) state with probability $1 / N_{h}$ and then determine the direction of the jump. It should be noted that we choose an occupied state simply by drawing a particle; hence, the probability that a state is chosen is proportional to the current number of particles occupying that state. The above procedure defines the most effective algorithm (since no Monte Carlo step is lost) which defines the relaxation process to the bosonic condensate.

The particles perform thermally activated jumps between the lattice sites since the lattice gas is coupled to a heat bath (canonical ensemble). To define the local dynamics the jump (transition) rates $\Gamma_{\uparrow}$ and $\Gamma_{\downarrow}$ are assumed which obey the so-called detailed balance condition for the jump rates: $\Gamma_{\downarrow} / \Gamma_{\uparrow}=\exp \left(\Delta \varepsilon / k_{B} T\right)$. In fact, this condition is necessary to prove that the classical, non-interacting particles obey the Boltzmann statistics. Since this condition does not uniquely define the jump rates we can assume their simplest form $\Gamma_{\downarrow \uparrow} \sim \exp \left( \pm \Delta \varepsilon / 2 k_{B} T\right)$ as energy levels are equally distant; this leads to the following jump (transition) probabilities: $p_{\downarrow \uparrow}=p_{0} \exp \left( \pm \Delta \varepsilon / 2 k_{B} T\right)$, where $p_{0}=\frac{1}{2} \frac{1}{\cosh \left(\Delta \varepsilon / 2 k_{B} T\right)}$ is the normalization factor. Of course, other choices of jump rates (obeying a detailed balance condition) have no influence on the equilibrium quantities; they could play a role when one would study dynamic properties, e.g. relaxation in the system.

We assume the blocking boundary condition in the vertical direction although the length of the energetic ladder, $L$, and the ratio $\Delta \varepsilon / k_{B} T$ are limited so that in statistical equilibrium the macroscopic part of jumping particles, in principle, does not reach the top of the ladder, i.e. $\left(p_{\uparrow}\right)^{L} \ll p_{\downarrow}$.

\section{Results}

We observed that already the system including several hundreds of particles reproduces quite well the Bose-Eintein condensation or $\lambda$-transition. Hence, it is possible to obtain the condensation and the corresponding phase diagram in real time by the Monte Carlo simulation within the interactive Java applet, cf. Fig.1. where temporal distribution of bosons among different energy levels is also shown (e.g., for $k_{B} T / \Delta \varepsilon=4.8$ ) to directly show the condensation phenomenon (here below the relative transition temperature $k_{B} T_{C} / \Delta \varepsilon \approx 6.9$ ).

By using the applet we are able to calculate and show the characteristic thermodynamic quantities such as boson occupancies and fluctuations, internal energy, entropy and the specific heat of the bosonic system as a function of temperature. We are able to show (with good approximation) that internal energy and entropy are continuous but non-differentiable functions of the relative temperature $k_{B} T / \Delta \varepsilon$ at the transition temperature. Hence, we show that the specific heat has characteristic discontinuity at this temperature which is a 


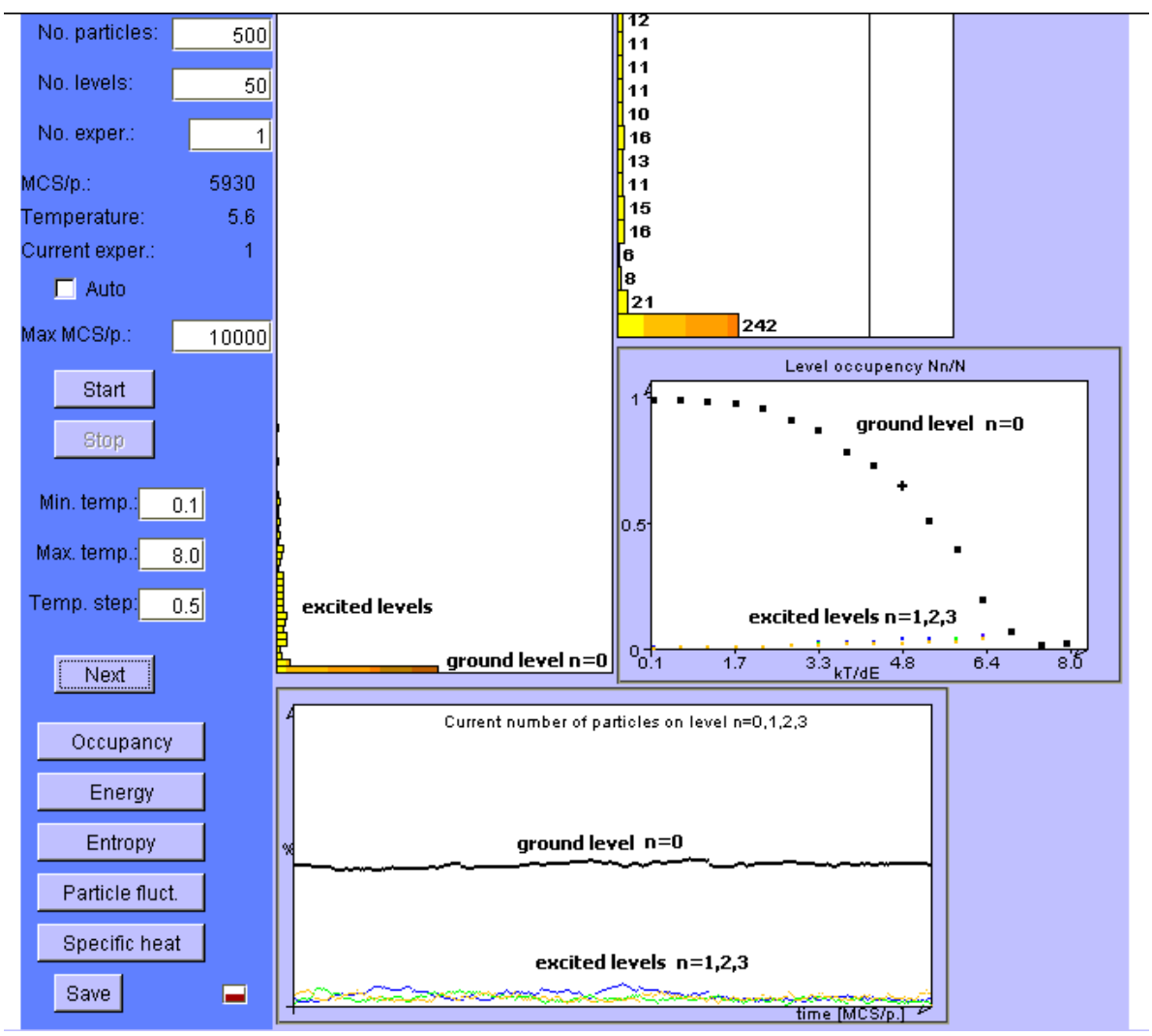

Fig. 1. Screen's picture given by our applet: (i) the phase diagram of the Bose-Einstein condensate (denoted by large black squares shown in the plot placed in the middle right window) i.e. the equilibrium ground-level occupancy $N_{0} / N$ vs. relative temperature, $k_{B} T / \Delta \varepsilon$, for example, for $N=500$ bosons in the system obtained in real time by our interactive Java applet (within a single experiment but with sufficiently good accuracy). (ii) The equilibrium distribution of bosons among energy levels obtained after a sufficiently long time (counted by Monte Carlo steps/particle) is shown (by horizontal indicators) in the diagram placed in the left middle window; its main part, including occupancies of the first 15 energy levels, is placed in the upper window. (iii) The lower window presents occupancies of the ground-level (the highest plotted curve) and three consecutive excited levels versus time (curves at the bottom); in this simple way we make it clear when the system reaches the statistical equilibrium, i.e. when there are no trends in the time-dependences. Temporal distributions considered in (ii) and (iii) relate to the condensate, for example, in the macrostate denoted in the phase diagram by plus $(+)$. 
clear finger print of $\lambda$-type phase transition. Moreover, there is no latent heat of the phase transition and therefore we can conclude that it is not of the first order. Note the pronounced $\lambda$-shape of the curve reminds the specific heat curve of $\mathrm{He}^{4}$ near the transition to superfluidity.

\section{Concluding Remarks}

An algorithm is presented and its possibilities are discussed for studying the condensation phenomenon occurring within the bosonic lattice gas. Below the transintion temperature we observe the physical phenomenon characteristic for the macroscale as the macroscopic amount of bosons occupies the ground level in distinction from the situation where the temperature exceeds the transition one; then, a microscopic amount of bosons occupy each energy level. Applying our Monte Carlo simulations we are able to study statistical physics and thermodynamics of the Bose-Einstein condensate which could also have meaning for studying similar aspects of superfluidity. The approach well illustrates the properties of the bosonic lattice gas and can help in understanding the open problems concerning, e.g., the dynamical behavior of bosonic systems. Our approach is promising also for education since already small systems consisting of a few hundreds of lattice bosons, reproduces quite well the characteristic properties of macroscopic systems.

\section{References}

1. Landau D.P., Binder K., A Guide to Monte Carlo Simulations in Statistical Physics. Springer-Verlag, Berlin (2000)

2. Anderson M.H., Ensher J.R., Matthews M.R., Wieman C.E., Cornell E.A.: Observation of Bose-Einstein Condensation in a Dilute Atomic Vapor. Science 269 (1995) 198-201.

3. Townsend Ch., Ketterle W., Stringari S.: Bose-Einstein condensation. Physics World 10 No 3 (1997) 29-34.

4. Bardou F., Bouchaud J.-P., Aspect A., Cohen-Tannaoudji C.: Lévy Statistics and Laser Cooling. How Rare Events Bring Atoms to Rest. Cambridge Univ. Press, Cambridge (2002)

5. Kutner R., Regulski M.: Bose-Einstein condensation shown by Monte Carlo simulation. Comp. Physics Comm. 121-122 (1999) 586-590

6. Wilkens M., Weiss Ch.: Particle number fluctuations in an ideal Bose gas. J. Mod. Optics 44 (1997) 1801-1814.

7. Navez P., Bitouk D., Gajda M., Idziaszek Z., Rzążewski K.: Fourth Statistical Ensemble for the Bose-Einstein Condensate. Phys. Rev. Lett. 79 (1997) 1789-1792.

8. Reif F.: Fundamentals of Statistical and Thermal Physics. McGraw-Hill, New York (1965)

9. Gould H., Spornick L., J. Tobochnik J.: Thermal and Statistical Physics Simulations. CULPS. J. Wiley \& Sons, New York (1995)

10. Kutner R., Kehr K.W., Renz W., Przeniosło R.: Diffusion in a one-dimensional bosonic lattice gas. J. Phys. A: Math. and General 28 (1995) 923-940. 